\title{
Experimental study on penetration of dental implants into the maxillary sinus in different depths
}

\author{
Weijian ZHONG, Binke CHEN, Xin LIANG, Guowu MA
}

Department of Oral Maxillofacial Surgery, College of Stomatology, Dalian Medical University, Dalian, China.

Corresponding address: Guowu Ma - No. 9 West part of Lvshun Southern Road - College of Stomatology, Dalian Medical University - Dalian 116044 - China - Phone: 0411-86110398 - Fax: 0411-86110398 - e-mail: mgw640242000@yahoo.com.cn

Submitted: January 15, 2013 - Modification: June 19, 2013 - Accepted: August 23, 2013

\section{ABSTRACT}

\begin{abstract}
$T^{\mathrm{h}}$ he exposing of dental implant into the maxillary sinus combined with membrane perforation might increase risks of implant failure and sinus complications. Objective: The purpose of this study was to investigate the effects of the dental implant penetration into the maxillary sinus cavity in different depths on osseointegration and sinus health in a dog model. Material and Methods: Sixteen titanium implants were placed in the bilateral maxillary molar areas of eight adult mongrel dogs, which were randomly divided into four groups according to the different penetrating extents of implants into the sinus cavities (group A: $0 \mathrm{~mm}$; group B: $1 \mathrm{~mm}$; group C: $2 \mathrm{~mm}$; group D: $3 \mathrm{~mm}$ ). The block biopsies were harvested five months after surgery and evaluated by radiographic observation and histological analysis. Results: No signs of inflammatory reactions were observed in any maxillary sinus of the eight dogs. The tips of the implants with penetrating depth of $1 \mathrm{~mm}$ and $2 \mathrm{~mm}$ were found to be fully covered with newly formed membrane and partially with new bone. The tips of the implants with penetrating depth over $3 \mathrm{~mm}$ were exposed in the sinus cavity and showed no membrane or bone coverage. No significant differences were found among groups regarding implant stability, bone-to-implant contact (BIC) and bone area in the implant threads (BA). Conclusions: Despite the protrusion extents, penetration of dental implant into the maxillary sinus with membrane perforation does not compromise the sinus health and the implant osseointegration in canine.
\end{abstract}

Key words: Maxillary sinus. Dental implants. Complications.

\section{INTRODUCTION}

While a limited amount of bone is generally present at the edentulous posterior maxilla due to atrophy of alveolar ridge and pneumatization of the maxillary sinus, several techniques have been described in which dental implants are inserted marginally to the bony sinus floor with a localized augmentation procedure ${ }^{3,18}$. Since these approaches have become conventional treatments in Implant Dentistry, the risk of exposing the implant to the maxillary sinus increased. The incidence of the sinus membrane perforation was reported as $7 \%$ to $35 \% 5,16,17$, and the sinus lifting procedure was abandoned in some studies because of the large perforation of the sinus membrane ${ }^{10,21}$.

In general, the sinus membrane perforation is considered as a potential risk factor for implant failure and sinus infection. Some investigators claimed that the membrane perforation was strongly associated with the occurrence of postoperative sinus infection ${ }^{6,22}$, while others assumed that there was a correlation between implant failure and sinus membrane perforation ${ }^{8,11,13}$. However, clinicians have generally reported that slight membrane perforation after implant placement does not play a significant role in the clinical outcome ${ }^{4,15,19}$. Nevertheless, the available literature has not conclusively determined so far the significance of implant exposure to the sinus cavity on implant survival and maxillary sinus complication, particularly with respect to the histological evidences.

Because most reported results of the sinus membrane perforation are clinical observations, 
they lack well-defined outcome criteria or control. In order to help clinicians to make proper surgical decisions, data on a more controlled scientific level is necessary to be provided.

The purpose of the present study was to evaluate the effects of dental implant exposure to maxillary sinus cavity with penetrating depth of $1-3 \mathrm{~mm}$ on osseointegration and sinus health in a dog model after a 5-months healing period.

\section{MATERIAL AND METHODS}

\section{Animals}

Eight healthy adult male mongrel dogs (weight 14.8-19.5 kg, age 20-24 months old) without any oral and systemic diseases were included in this study. The animals were housed individually in indoor cages and the diet during the course of the experiment was whole grain flour, cornmeal, soybean cake, fishbone meal, and eggs, according to the general feeding program at Experimental Animal Center of Dalian Medical University, China. The protocol for this animal study was approved by the Ethics Committee of Dalian Medical University (protocol number: 2007-05A).

\section{Experimental groups}

A split-mouth randomized design, using four treatment protocols on the positions of bilateral maxillary first molars, was employed. In total, 16 samples were used in this study. Each implant recipient site was randomly assigned to one of the four treatment protocols, and immediate implant placement was applied accordingly: (1) group A $(n=4)$, was control group without sinus floor penetration; (2) group $B(n=4)$, with penetrating depth of $1 \mathrm{~mm}$; (3) group $C(n=4)$, with penetrating depth of $2 \mathrm{~mm}$; (4) group $D(n=4)$, with penetrating depth of $3 \mathrm{~mm}$. The investigators ensured unpredictability of the allocation sequence by coin toss before surgeries.

\section{Surgical procedures}

The dogs received 1 week of antibiotics prophylaxis (gentamicin sulfate, $16000000 \mathrm{U} /$ day, Linggui Pharmaceutical Co.Ltd, Zhengzhou, China) to prevent infection. For all the surgical procedures involved in this study, animals were anesthetized intramuscularly with ketamine hydrochloride (10 $\mathrm{mg} / \mathrm{kg}$, Changchun Academy of Military Medical Sciences, Changchun, China). The anesthesia was supplemented by local administration of $2 \%$ lidocaine HCI with $1: 100,000$ epinephrine (2-4) $\mathrm{ml}$, Shandong Pharmaceutical Production Ltd, Shandong, China). The whole surgical procedure was performed under sterile conditions by only one surgeon (W.Z.), with a decade of clinical experience in Implant Dentistry.
After the maxillary first molars were extracted bilaterally with special care, immediate implant placements were performed in the palatal sockets. Sixteen custom-made threaded cylindrical implants of grade 5 pure titanium $(\varnothing=3.75 \mathrm{~mm}, \mathrm{~L}=10 \mathrm{~mm}$, CDIC, Sichuan University, China) were thoroughly examined and sterilized prior to surgery. Each site was prepared with twist drills at low speed of 500 $\mathrm{rpm}$ while being cooled with sterile saline at $4^{\circ} \mathrm{C}$. After the bone and mucous membrane of the sinus floors were intentionally drilled through, the level of the sinus floor was detected with a dental probe and the height of residual ridge was measured. Except for the implants of control group placed within the alveolar bone without protruding into the sinus cavity, the other twelve implants were placed bilaterally in the sinus in such a way that they penetrated the bone and mucous membrane of the maxillary sinus floor to the extent of $1 \mathrm{~mm}, 2 \mathrm{~mm}$, or $3 \mathrm{~mm}$, respectively. The buccal mucoperiosteal flaps were released and sutured with 4-0 vicryl resorbable sutures.

To prevent postoperative infections, the animals received a daily penicillin intramuscular injection ( 2 doses of 100,000 units/kg, Penicillin G sodium, Shandong Pharmaceutical Production Ltd, Shandong, China) for one week, and plaque control was ensured three times per week using a $0.2 \%$ chlorhexidine gel on implant placement sites with soft toothbrush. A soft diet was given to the animals during the healing period.

\section{Resonance frequency analysis}

The stability of the implants was assessed with RFA (Osstell ${ }^{\mathrm{TM}}$, Integration Diagnostics $A B$, Goteborg, Sweden) in implant stability quotient units at the time of implant placement and 5 months later.

\section{Sacrifice and samples preparation}

Five months after surgery, the animals were sacrificed with overdose pentobarbital sodium and vascular perfusion was undertaken with paraformaldehyde. The sample blocks of maxilla with implants were harvested and the upper walls of the sinus were eliminated to expose the sinus floor. The specimens were trimmed and immersed in $4 \%$ paraformaldehyde in $0.1 \mathrm{M}$ sodium phosphate buffer.

\section{Observation and histological assessment}

After a gross observation, radiographic examination was performed with periapical radiograph using paralleling technique, and the $X$-ray beam was perpendicular to the long axis of the implant and film. All samples were shot using the following exposure conditions: $70 \mathrm{Kv}$ tube voltage, $10 \mathrm{~mA}$ tube current and 0.32-second exposing time. 
Thereafter, the membrane adjacent to the implant was processed into paraffin section and stained with hematoxylin and eosin.

After dissection of the jawbones, each implant with a preserved 5-mm thickness of peri-implant bone was removed as one piece of sample. Following dehydration in a series of ascending grades of ethanol, the bone blocks were embedded in hardgrade acrylic resin (LR WhiteTM, London Resin Company Ltd, Berkshire, England) and polymerized in a dry heat oven at $60^{\circ} \mathrm{C}$. Blocks were cut longitudinally, sections were ground to a thickness of $30 \mu \mathrm{m}$ and stained with methylene blue and aniline red (Exact Cutting and Grinding equipment, Exact Apparatebau, Norstedt, Germany). Three coronal sections from central area of each implant were obtained for histological assessment. Histomorphometric analysis of BIC and BA were performed using Scion Image software (NIH, Bethesda, MD, USA).

\section{Statistical analysis}

The results of BIC and BA were recorded as mean value (standard deviation) from the twelve sections of each group. Statistical analysis of differences between groups was performed using one-way ANOVA with SPSS 11.0 software (SPSS Inc., Chicago, IL, USA). Probability (P) values less than 0.05 were considered significant.

\section{RESULTS}

At the time of surgical procedure, the animals showed no clinical signs of any sinus disorder. The postoperative healing was uneventful in all of the cases. All animals recovered rapidly from surgeries and were healthy throughout the follow-up period. There was not any sign of wound infection or other complications such as implant loosening or falling. Two cover screws were exposed in the control group.

\section{Resonance frequency analysis}

In all groups, primary stability was obtained and maintained to the time of sacrifice. The values from implant stability quotient measurements were recorded at the moment of implants placement and animal sacrifice (Table 1). No apparent differences were found among groups.

\section{Gross examination}

The bone and membrane of sinus floor remained intact in the control group (Figure $1 \mathrm{a}$ ). In group $B$ and group $\mathrm{C}$, the protruding parts of implants that had been introduced into the sinus cavity for $1 \mathrm{~mm}$ and $2 \mathrm{~mm}$ were fully covered with a thin layer of newly formed membrane with a healthy appearance (Figures 1b, 1c). In group $D$, the parts of implants that had been penetrated into the sinus cavity for $3 \mathrm{~mm}$ were totally exposed in the sinus cavity with the membrane surrounding the base of protruding parts (Figure 1d). No signs of inflammation were observed in the sinus membranes of all samples.

\section{Radiographic examination}

Radiographic examination of the sites of implantation demonstrated a close contact of bone and implants without radiolucent areas. The tips of implants in the control group were completely embedded in the alveolar bone (Figure 2a). The tips of implants with the penetrating depth of $1 \mathrm{~mm}$ and $2 \mathrm{~mm}$ were surrounded by bone tissue (Figures 2b,

Table 1- Implant stability quotient values [Mean(SD)] measured by resonance frequency analysis at the time of implant placement and animal sacrifice

\begin{tabular}{ccccc}
\hline ISQ value & \multicolumn{3}{c}{ Groups } \\
\hline A & B & C & D \\
\hline Sacement & $66.1(4.4)$ & $65.5(3.2)$ & $63.8(4.8)$ & $68(9.6)$ \\
\hline
\end{tabular}

ISQ: Implant stability quotient

SD: Standard Deviation

Table 2- Comparison of the percentages of bone-implant contact and bone area in threads [Mean(SD)] among groups

\begin{tabular}{ccccc}
\hline Percentage & \multicolumn{3}{c}{ Groups } \\
& A & B & C & D \\
\hline BIC & $19.2(12.1)$ & $15.5(8.9)$ & $16.8(14.4)$ & $10.3(11.6)$ \\
BA & $34.7(21.3)$ & $28.2(17.5)$ & $32.2(24.5)$ & $16.5(12.2)$ \\
\hline
\end{tabular}

BIC: Bone-implant contact; BA: Bone area in threads

SD: Standard Deviation 
2c). The tips of implants with the penetrating depth of $3 \mathrm{~mm}$ were found to protrude inside the sinus cavity without bone coverage (Figure $2 \mathrm{~d}$ ).

\section{Histological examination}

The sinus membrane presented no discernible inflammatory reactions in any of the maxillary sinus cavities. The thickening epithelial lamina and increasing amount of fibrous tissue in the lamina propria that was arranged in derangement were observed in the membrane at the site of perforation. This indicated that the wound healing process had occurred at this part of the membrane. In group $D$, the apical portion of totally exposed implant was surrounded by respiratory epithelium without inflammatory cells infiltration (Figure 3).
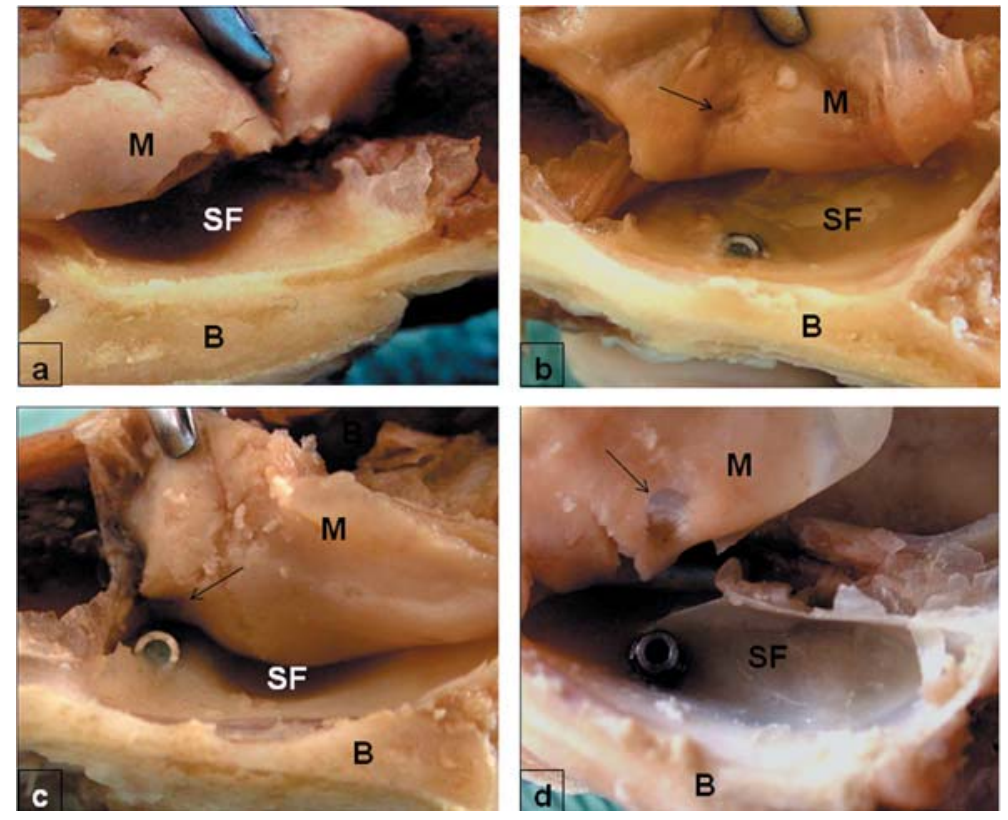

Figure 1- Gross observation of the apical portion of implant in sinus cavity. The bony sinus floor in the control group (a) remained intact without implant exposure. The tips with protruding depth of $1 \mathrm{~mm}$ (b) and $2 \mathrm{~mm}$ (c) were partially covered with new bone and scars were left at the penetrating spots on the membrane (arrows). The implant tip with protruding depth of $3 \mathrm{~mm}$ (d) was totally exposed in the sinus cavity with no bone coverage and the penetrating hole was left on the sinus membrane (arrow). SF: sinus floor; M: sinus membrane; B: bone of lateral sinus wall
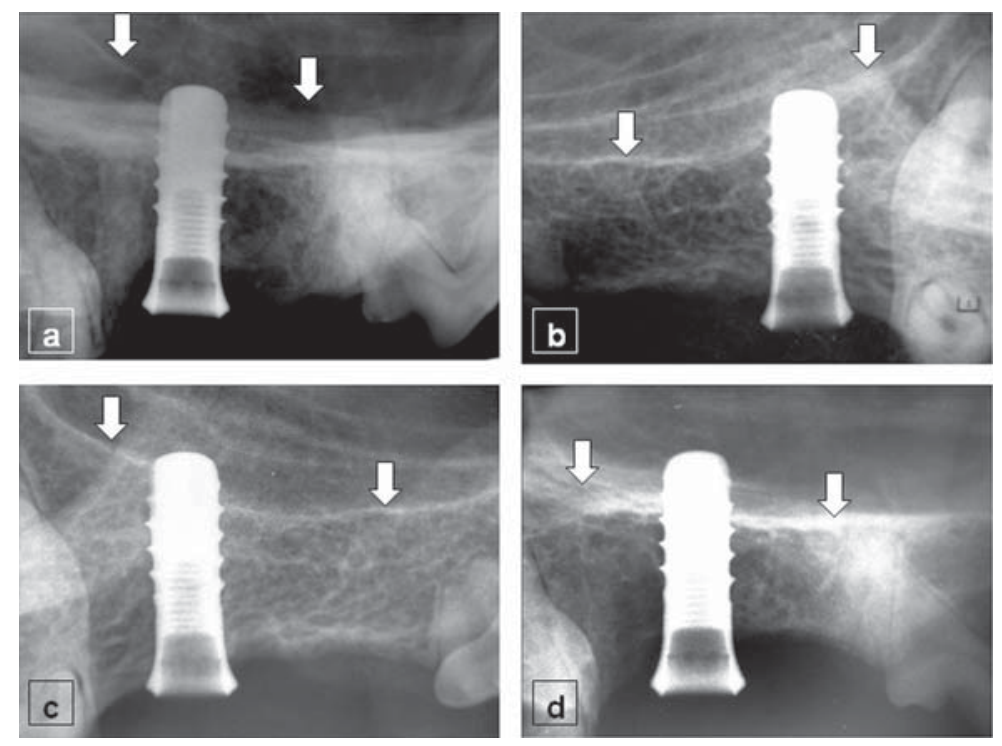

Figure 2- Radiographic examination of the sites of implantation. The tips of implants in the control group (a) were completely embedded in the alveolar bone. The tips of implants with penetrating depth of $1 \mathrm{~mm}$ (b) and $2 \mathrm{~mm}$ (c) were surrounded by bone tissue. The tip of implant with the penetration depth of $3 \mathrm{~mm}$ was found to protrude inside the sinus cavity with no bone coverage (d). Arrows indicate the bony sinus floor 


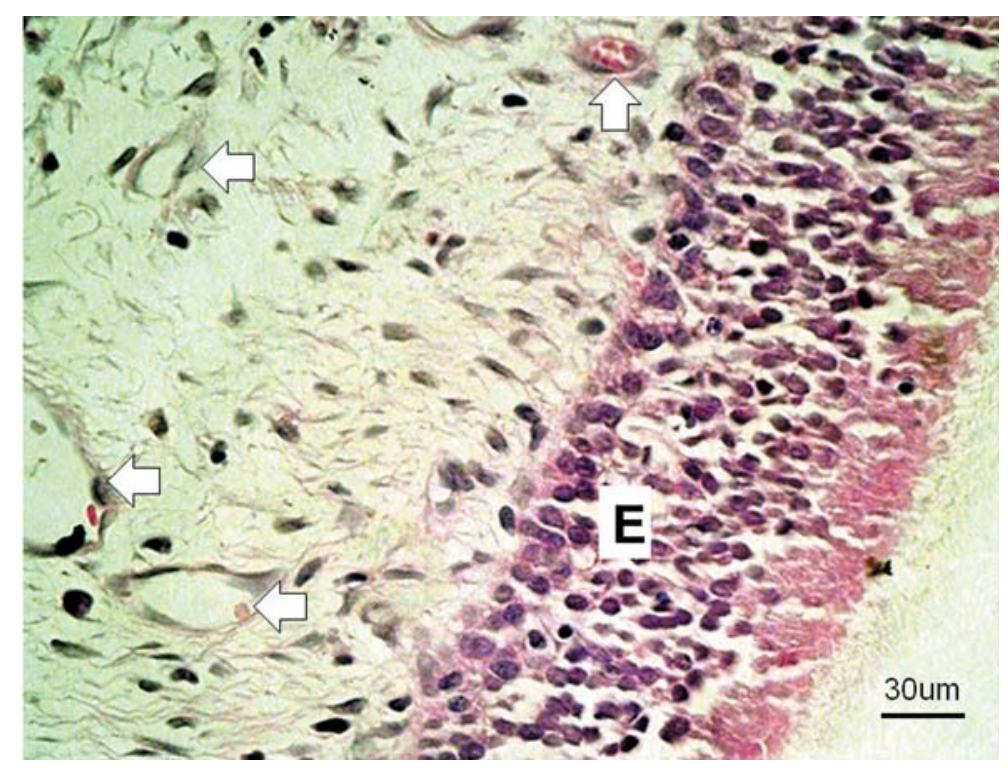

Figure 3- Histological micrograph of the membrane surrounding the protruding portion of implant in group D. Abundant blood vessels (arrows) were found in the lamina propria with no inflammatory cells infiltration. E: epithelium. (Hematoxylin and eosin)

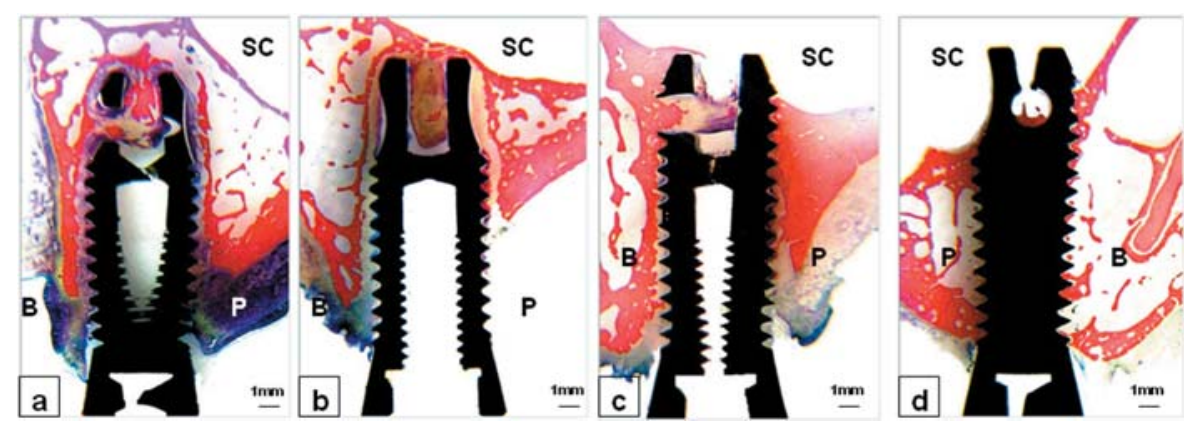

Figure 4- Typical histological micrographs of the coronal plane ground sections of the control group (a), group B (b), group C (c), and group D (d). SC: sinus cavity; B: buccal side; P: palatal side. (methylene blue and aniline red)

Ground sections of bone blocks showed a successful osseointegration between all implants and bony sinus floor, and the growing bone tissue was observed in the retention hole of the implant which indicated an ongoing process of bone regeneration. Although a perfect osseointegration had not been achieved at the coronal parts in the control group, where a thin layer of fibrous tissue was present at bone-implant interface, the apical portions of all implants in this group were completely embraced by newly formed bone (Figure 4a). In all the test groups, the apical parts of the implants became integrated with the bony sinus floor that was mostly lamellar bone, whereas the mid-portions were surrounded by trabecular bone and medullary spaces. Although the coronal portions of the implants in group B were surrounded with fibrous connective tissue, the apical parts were completely covered by new bone tissue (Figure 4b). The uppermost parts of the implants in group $C$ were partially covered with regenerated bone tissue (Figure 4c). However, the apical parts of implants in group $D$ that had protruded into the sinus cavity for $3 \mathrm{~mm}$ were not covered with bone tissue, but perfect osseointegration was achieved without fibrous tissue formation in the interface (Figure 4d).

\section{Histomorphometric analysis}

The mean values of BIC and BA are displayed in Table 2. Statistical analysis revealed that no significant differences in the BIC and BA were present among groups $(P>0.05)$, although the relatively lower BIC and BA values were observed in the control group.

\section{DISCUSSION}

It is generally acknowledged that the implant displaced into the maxillary sinus can act as a foreign body and thus can cause serious ongoing complications $^{7,9}$. It has also been reported that implant penetration into sinus cavity resulted in recurrent rhino-sinusitis ${ }^{14}$. The most likely explanation for this complication is that altered 
nasal airflow could induce irritation of the nasal mucosa ${ }^{12}$. In addition, nasal clearance could be disturbed by implant blockage of the mucociliary pathway giving rise to inflammation ${ }^{23}$. However, evidence is scarce concerning whether the length of implants penetration into the sinus cavity might inhibit the spontaneous recovery of membrane perforation and further result in development of rhino-sinusitis.

The present animal experiment was designed to evaluate whether there are differences between minor and major penetrations of implants into maxillary sinus, with respect to their effect on implant osseointegration and sinus complication. Despite the different penetrating depths, the osseointegration in the interface between implant and bony sinus floor was achieved, and no inflammatory reaction was observed in the surrounding sinus membrane, suggesting that the exposed implants do not make the maxillary sinus membrane vulnerable to complications.

Disruptive membrane around the apical portion of implant healed again and re-covered the tips of implants provided the protruding depths were less than $2 \mathrm{~mm}$. Regenerated bone tissue was discovered on the uppermost part of some implants which indicated the self-regenerating and new bone-inducing abilities of sinus membrane. Based on the present result, it seems that under the circumstance of everyday practice, it is relatively safe to control the implant protrusion depth to the extent of less than $2 \mathrm{~mm}$ in case a healthy maxillary sinus was accidentally perforated.

Whereas, when the protruding depth was deeper than $3 \mathrm{~mm}$, membrane coverage of the exposed portion could not be achieved. Circular epithelium structure similar to gingival cuff formed around the base of the protruding parts and did not show any sign of inflammation. This observation may be explained by the direct attachment of the membrane to the implants, forming a barrier to the sinus cavity. As for the parts that were not covered with the sinus membrane, debris might accumulate on the surface of the exposed apical part over time and become a potential predisposition to sinusitis.

It was reported that transient sinusitis was prone to develop in patients with a predisposition for sinusitis ${ }^{19}$. Unhealthy sinus with chronic inflammation or cyst combined with membrane perforation may lead to severe acute sinus infection. It is prudent to evaluate all patients preoperatively by intranasal examination to rule out those individuals with a history of sinus pathology that could be aggravated by inflammation associated with the sinus lift procedure. For implants that were accidentally penetrated through the sinus membrane and exposed into the sinus cavity, provided that the sinus was healthy before operation and necessary postoperative antiinfection management was given, these implants were suggested to remain in place for follow-up observation ${ }^{1,2,20}$. But more clinical evidences were needed to confirm this standpoint.

Because the immediate implant placement after extraction was performed in this study and no bone grafting or any membrane technique was used, it was accessible that the poorly embedded cervical portions of some implants in group A and $B$ were encapsulated with fibrous tissue resulting in relatively lower BIC and BA values. But the clinical stability of these implants was achieved and the apical osseointegration was confirmed by histological analysis.

Although the 5-months observational period was too short and an animal model cannot exactly replicate the human environment, such studies may provide the best possible answers to the problems relating implants exposed to the sinus cavity. However, further investigations are necessary to evaluate long term effects of penetrating implant loaded with prosthesis or combined with bone graft.

\section{CONCLUSIONS}

Within the limits of this study, we can conclude that, despite the different protrusion extents, penetration of dental implants into the maxillary sinus with membrane perforation does not compromise the implant osseointegration and the sinus health during the 5-months observational period in canines. When the penetrating depth into the sinus is less than $2 \mathrm{~mm}$, the apical portion of implant could be re-covered by regenerating membrane.

\section{REFERENCES}

1- Aimetti M, Romagnoli R, Ricci G, Massei G. Maxillary sinus elevation: the effect of macrolacerations and microlacerations of the sinus membrane as determined by endoscopy. Int J Periodontics Restorative Dent. 2001;21:581-9.

2- Berengo M, Sivolella S, Majzoub Z, Cordioli G. Endoscopic evaluation of the bone-added osteotome sinus floor elevation procedure. Int J Oral Maxillofac Surg. 2004;33:189-94.

3- Boyne PJ, James RA. Grafting of the maxillary sinus floor with autogenous marrow and bone. J Oral Surg. 1980;30:613-6.

4- Brånemark PI, Adell R, Albrektsson T, Lekholm U, Lindström J, Rockler A. An experimental and clinical study of osseointegrated implants penetrating the nasal cavity and maxillary sinus. J Oral Maxillofac Surg. 1984;42:497-505.

5- Chanavaz M. Maxillary sinus: anatomy, physiology, surgery, and bone grafting related to implantology - eleven years of surgical experience (1979-1990). J Oral Implantol. 1990;16:199-209. 6- Cordioli G, Mazzocco C, Schepers E, Brugnolo E, Majzoub Z. Maxillary sinus floor augmentation using bioactive glass granules and autogenous bone with simultaneous implant placement. Clinical and histological findings. Clin Oral Implants Res. 2001;12:270-8. 
7- Givol N, Taicher S, Halamish-Shant T, Chaushu G. Risk management aspects of implant dentistry. Int J Oral Maxillofac Implants. 2002;17:258-62.

8- Hernández-Alfaro F, Torradeflot MM, Marti C. Prevalence and management of Schneiderian membrane perforations during sinuslift procedures. Clin Oral Implants Res. 2008;19:91-8.

9- Iida S, Tanaka M, Kogo M, Matsuya T. Migration of a dental implant into the maxillary sinus. A case report. Int J Oral Maxillofac Surg. 2000;29:358-9.

10- Karabuda C, Arisan V, Özyuvaci H. Effects of sinus membrane perforations on the success of dental implants placed in the augmented sinus. J Periodontol. 2006;77:1991-7.

11- Khoury F. Augmentation of the sinus floor with mandibular bone blocks and simultaneous implantation: a 6-year clinical investigation. Int J Oral Maxillofac Implants. 1999;14:557-64.

12- Levin L, Herzberg R, Dolev E, Schwartz-Arad D. Smoking and complications of onlay bone grafts and sinus lift operations. Int J Oral Maxillofac Surg. 2004;19:369-73.

13- Proussaefs P, Lozada J, Kim J, Rohrer MD. Repair of the perforated sinus membrane with a resorbable collagen membrane: a human study. Int J Oral Maxillofac Surg. 2004;19:413-20. 14- Raghoebar GM, Weissenbruch R, Vissink A. Rhino-sinusitis related to endosseous implants extending into the nasal cavity. A case report. Int J Oral Maxillofac Surg. 2004;33:312-4.

15- Reiser GM, Rabinovitz Z, Bruno J, Damoulis PD, Griffin TJ. Evaluation of maxillary sinus membrane response following elevation with the crestal osteotome technique in human cadavers. Int J Oral Maxillofac Implants. 2001;16:833-40
16- Schwartz-Arad, D, Herzberg, R, Dolev E. The prevalence of surgical complications of the sinus graft procedure and their impact on implant survival. J Periodontol. 2004;75:511-6.

17- Shlomi B, Horowitz I, Kahn A, Dobriyan A, Chaushu G. The effect of sinus membrane perforation and repair with Lambone on the outcome of maxillary sinus floor augmentation: a radiographic assessment. Int J Oral Maxillofac Implants. 2004;19:559-62.

18-Tatum H Jr. Maxillary and sinus implant reconstruction. Dent Clin North Am. 1986;30:207-29.

19- Timmenga NM, Raghoebar GM, Boering G, van Weissenbruch R. Maxillary sinus function after sinus lifts for the insertion of dental implants. J Oral Maxillofac Surg. 1997;55:936-9.

20- Timmenga NM, Raghoebar GM, van Weissenbruch R, Vissink A. Maxillary sinus floor elevation surgery. A clinical, radiographic and endoscopic evaluation. Clin Oral Implants Res. 2003;14:322-8. 21- Valentini P, Abensur DJ. Maxillary sinus grafting with anorganic bovine bone: a clinical report of long-term results. Int J Oral Maxillofac Implants. 2003;18:556-60.

22- Van den Bergj JP, ten Ruggenkate CM, Disch FJ, Tuinzing DB. Anatomical aspects of sinus floor elevations. Clin Oral Implants Res. 2000;11:256-65.

23- Watelet JB, Bachert C, Gevaert P, Cauwenberge P. Wound healing of the nasal and paranasal mucosa: a review. Am J Rhinol. 2002; 16:77-84. 